PROCEEDINGS OF THE

AMERICAN MATHEMATICAL SOCIETY

Volume 126, Number 11, November 1998, Pages 3393-3396

S 0002-9939(98)04956-9

\title{
ON THE HYPERSPACE OF A NON-SEPARABLE METRIC SPACE
}

\author{
CAMILLO COSTANTINI
}

(Communicated by Franklin D. Tall)

\begin{abstract}
We prove the following two results.
1) There exists a non-separable complete metric space whose Wijsman hypertopology is not Čech-complete.

2) There exist a non-separable metrizable space and two compatible metrics on it, such that the collections of the Borel sets generated by the relative Wijsman hypertopologies do not coincide.
\end{abstract}

\section{INTRODUCTION}

The main purpose of this paper is to underline the differences between the hyperspace of a non-separable metric space and that of a separable metric space, with special regard to the Wijsman hypertopology (for general reference, see [Be1, $\S \S 2.1$ and 3.2]).

In the literature, the separable case is by far the best studied, and has proved to have interesting applications to analysis, measure theory and descriptive set theory (cf., for example, $[\mathrm{Be} 2, \S 1]$ and $[\mathrm{Ke}, \S 12 . \mathrm{C}]$ ). We will present here two counterexamples showing that two classical results about the hyperspace of a separable metric space do not extend to the non-separable case.

\section{First EXAmple}

If $(X, d)$ is a separable and complete metric space, then the Wijsman topology $\mathbf{w}_{d}$ on $c_{o}(X)$ (see definition below) is in turn separable and completely metrizable [Be2, Theorem 4.3]. This result can be generalized by showing that for every separable and completely metrizable space $X$ and for every compatible metric $d$ on $X$, the space $\left(c_{o}(X), \mathbf{w}_{d}\right)$ is still separable and completely metrizable [Co] (note that such a generalization is not automatic, as equivalent metrics on a set $X$ can give rise to different Wijsman topologies on $c_{o}(X)$ - cf., in particular, [CLZ, Theorem $5^{\prime}$ ]).

If the metric space $(X, d)$ is not separable, then $\left(c_{o}(X), \mathbf{w}_{d}\right)$ is neither separable nor metrizable, as each of these properties is in fact equivalent to the separability of the base space (cf. [Be1, Theorem 2.1.5] and related bibliography). However, it is worth wondering whether the complete metrizability of $(X, d)$, or at least its actual completeness, can imply some suitable form of completeness for $\left(c_{o}(X), \mathbf{w}_{d}\right)$.

Received by the editors January 17, 1996 and, in revised form, November 18, 1996.

1991 Mathematics Subject Classification. Primary 54B20; Secondary 54E35, 54D35.

Key words and phrases. Non-separable metric space, Wijsman hypertopology, Čech-completeness, Borel sets.

(C)1998 American Mathematical Society 
As a natural candidate, we will consider Čech-completeness, which in the case of a metrizable space is equivalent to complete metrizability.

In the following, we give a negative answer to the above question, by exhibiting a (non-separable) complete metric space $(X, d)$ for which $\left(c_{o}(X), \mathbf{w}_{d}\right)$ is not Čechcomplete.

Let $(X, d)$ be a metric space. For every $x \in X$ and $\varepsilon>0$, we put:

$$
\mathcal{A}_{d}^{+}(x, \varepsilon)=\left\{C \in c_{o}(X) \mid d(x, C)>\varepsilon\right\}
$$

and

$$
\mathcal{A}_{d}^{-}(x, \varepsilon)=\left\{C \in c_{o}(X) \mid d(x, C)<\varepsilon\right\} .
$$

The Wijsman topology $\mathbf{w}_{d}$ on $c_{o}(X)$ is that having as a subbase the collection:

$$
\Delta_{d}=\left\{\mathcal{A}_{d}^{+}(x, \varepsilon) \mid x \in X, \varepsilon>0\right\} \cup\left\{\mathcal{A}_{d}^{-}(x, \varepsilon) \mid x \in X, \varepsilon>0\right\} .
$$

Observe that if we associate to every $A \in c_{o}(X)$ the continuous function $\varphi(A)$ from $X$ to $\mathbf{R}$, defined by $(\varphi(A))(x)=d(x, A)$, then $\varphi$ is a topological embedding of $\left(c_{o}(X), \mathbf{w}_{d}\right)$ into $C(X)$, endowed with the topology of the pointwise convergence.

In the special case - which is often considered in this paper - where the metric $d$ takes its values on the three-element set $\{0,1,2\}$, we will also use the notation $[x / i]_{d}$, for $x \in X$ and $i \in\{0,1,2\}$, to denote the collection $\left\{A \in c_{o}(X) \mid d(x, A)=i\right\}$. Since in this case $\varphi$ is in fact an embedding of $\left(c_{o}(X), \mathbf{w}_{d}\right)$ into $3^{X}$, we have that the collection $\left\{[x / i]_{d} \mid x \in X, i \in\{0,1,2\}\right\}$ is a subbase of $\left(c_{o}(X), \mathbf{w}_{d}\right)$ (consisting of clopen sets).

Now, let $R$ be the real line endowed with the discrete topology. Let $\left\{A_{x} \mid x \in R\right\}$ be a listing of the collection of all countable subsets of $R$, such that for every $A \subseteq R$ with $|A| \leq \aleph_{0}$ we have that $\left|\left\{x \in R \mid A_{x}=A\right\}\right|=\mathbf{c}$ (where $\mathbf{c}$ is the cardinality of the continuum). Also, let $\alpha$ be a one-to-one function from $R$ onto $\mathbf{c}$, and define a compatible metric $d$ on $R$ by:

$$
d(x, y)= \begin{cases}0, & \text { if } x=y \\ 2, & \text { if either } \alpha(y)<\alpha(x) \text { and } y \in A_{x}, \text { or } \alpha(x)<\alpha(y) \text { and } x \in A_{y} \\ 1, & \text { otherwise }\end{cases}
$$

To show that $\left(c_{0}(R), \mathbf{w}_{d}\right)$ is not Cech-complete, it will suffice to prove that $\varphi\left(c_{0}(R)\right)$ is not a $G_{\delta}$-subset of its closure into $3^{R}$, which is clearly compact. Let $\mathcal{K}=\varphi\left(c_{0}(R)\right) \cap 2^{R}$ : for every countable (non-empty) subset $A$ of $R$, we have that $\varphi(A) \notin 2^{R}$. Indeed, the set $M=\left\{\alpha(x) \mid x \in R, A_{x}=A\right\}$ has cardinality c, thus it is cofinal in c and hence, choosing a $\bar{x} \in R$ with $A_{\bar{x}}=A$ and $\alpha(y)<$ $\alpha(\bar{x})$ for every $y \in A$, we have that $(\varphi(A))(\bar{x})=2$. As a consequence, $\mathcal{K} \subseteq$ $\left\{\varphi(A)|| A \mid>\aleph_{0}\right\}$ and $\mathcal{K} \subseteq\left\{f \in 2^{R}||\{x \in R \mid f(x)=0\} \mid>\aleph_{0}\right\}=2^{R} \backslash \Sigma(\mathbf{1})$, where $\Sigma(\mathbf{1})=\left\{f \in 2^{R}||\{x \in R \mid f(x) \neq 1\} \mid \leq \aleph_{0}\right\}$ is the $\Sigma$-product in $2^{R}$ with base point 1 - the function having constant value 1 (for general references on $\Sigma$-products, see [En, Problem 2.7.14]).

The fact that $\Sigma(\mathbf{1}) \cap \mathcal{K}=\emptyset$ implies that $\mathcal{K}$ is not a $G_{\delta}$-subset of $2^{R}$, because $\Sigma(\mathbf{1})$ meets every non-empty $G_{\delta}$-subset of $2^{R}$. Indeed, let $\left\{\mathcal{A}_{n} \mid n \in \mathbf{N}\right\}$ be a family of open subsets of $2^{R}$ whose intersection contains at least an element $g$ : for every $n \in \mathbf{N}$ we can find a finite $F_{n} \subseteq X$, and for every $x \in F_{n}$ an $i_{n}(x) \in 2$, such that $g \in\left\{f \in 2^{R}|f|_{F_{n}}=i_{n}\right\} \subseteq \mathcal{A}_{n}$. Then $i=\bigcup_{n \in \mathbf{N}} i_{n}$ is still a function, and its 
domain $D$ is countable; therefore if we extend $i$ to a function $f$ on $X$, taking on the value 1 on $X \backslash D$, we have that $f \in\left(\bigcap_{n \in \mathbf{N}} \mathcal{A}_{n}\right) \cap \Sigma(\mathbf{1})$.

As an obvious general result we have that, if a subset $Y$ of a topological space $X$ is a $G_{\delta}$-subset of its closure in $X$, and $Z$ is another subset of $X$ such that $Y \cap Z$ is dense in $Z$, then $Y \cap Z$ is a $G_{\delta}$-subset of $Z$. Thus, if we can prove that $\mathcal{K}=\varphi\left(c_{0}(R)\right) \cap 2^{R}$ is dense in $2^{R}$, we will obtain that $\varphi\left(c_{0}(R)\right)$ is not a $G_{\delta^{-}}$subset of its closure in $3^{R}$.

Consider in $2^{R}$ the $\Sigma$-product $\Sigma(\mathbf{0})$ (where $\mathbf{0}(x)=0$ for every $x \in R$ ): since $\Sigma(\mathbf{0})$ is dense in $2^{R}$, it will suffice to show that $\Sigma(\mathbf{0}) \subseteq \mathcal{K}$. Let $f \in \Sigma(\mathbf{0})$ and $A=\{x \in R \mid f(x)=0\}$ : we claim that $\varphi(A)=f$, that is, $d(x, A)=1$ for every $x \in R \backslash A$. Observe that, by the definition of $d$, for every fixed $\bar{x} \in R \backslash A$ we have that $d(\bar{x}, A)=2$ if and only if

$$
A \subseteq\left\{y \in R \mid \alpha(y)<\alpha(\bar{x}) \text { and } y \in A_{\bar{x}}\right\} \cup\left\{y \in R \mid \alpha(y)>\alpha(\bar{x}) \text { and } \bar{x} \in A_{y}\right\} .
$$

Let $B$ be any countable subset of $R \backslash\{\bar{x}\}$ and $M=\left\{y \in R \mid A_{y}=B\right\}$ : then $|M|=\mathbf{c}$, so that $|M \cap A|=\mathbf{c}$, too; since $M \cap A \cap\left\{y \in R \mid \alpha(y)>\alpha(\bar{x})\right.$ and $\left.\bar{x} \in A_{y}\right\}=\emptyset$ and $\mid\left\{y \in R \mid \alpha(y)<\alpha(\bar{x})\right.$ and $\left.\bar{x} \in A_{y}\right\} \mid<\mathbf{c},(\Delta)$ does not hold, i.e., $d(\bar{x}, A)=1$.

Let us observe that, by [Zs, Corollary 5.2], the above constructed space is Baire.

\section{SECOND EXAMPLE}

Let $X$ be a separable metrizable space, and $d, \rho$ two compatible metrics on $X$. As we have already recalled, the two topologies $\mathbf{w}_{d}$ and $\mathbf{w}_{\rho}$ on $c_{o}(X)$ may very well be different; nevertheless, if we consider the collections $\Xi_{d}$ and $\Xi_{\rho}$ of the Borel sets they respectively generate, then by a result of $\mathrm{C}$. Hess we have coincidence $[\mathrm{He}$, Proposition 3.1.1]. As a matter of fact, it turns out that the collections $\Xi_{d}$ and $\Xi_{\rho}$ both coincide with the Effros sigma algebra on $c_{o}(X)$ (see [Be1, Theorem 6.5.14] and related bibliography).

We will prove here that the hypothesis of separability for $(X, d)$ cannot be dropped in the above result.

Let $Q$ denote the set of rational numbers in the interval $] 0,2[$ and let $P$ denote the set of irrational numbers in the interval $] 1,2[$, endowed with the Euclidean topology. For $x \in P$, put $A_{x}=\{q \in Q \mid 1 / x<q<x\}$, and let $\mathcal{R}=\left\{A_{x} \mid x \in P\right\}$. Clearly, the family $\mathcal{R}$ is incomparable, in the sense that for $x, y \in P$ with $x \neq y$, we have that $A_{x} \nsubseteq A_{y}$ and $A_{y} \nsubseteq A_{x}$.

First, consider the 0-1 metric $d$ on $X=Q \cup P$; we claim that $x \mapsto A_{x}$ is a homeomorphism between $P$ and $\mathcal{R}$, where $\mathcal{R}$ is endowed with the topology induced by $\left(c_{0}(Q \cup P), \mathbf{w}_{d}\right)$. Indeed, for every $q \in Q$ and $x \in P$ we have that $d\left(q, A_{x}\right)=0$ if and only if $x>q$ and $q>1 / x$; it follows that:

$$
\begin{gathered}
{[q / 0]_{d} \cap \mathcal{R}=\left\{A_{x} \mid x \in P \cap\right] q, 2[\cap] \frac{1}{q}, 2[\},} \\
{[q / 1]_{d} \cap \mathcal{R}=\left\{A_{x} \mid x \in P \cap(] 1, q[\cup] 1, \frac{1}{q}[)\right\}}
\end{gathered}
$$

(of course, if $q \in P$, then $[q / 0]_{d} \cap \mathcal{R}=\emptyset$ and $[q / 1]_{d} \cap \mathcal{R}=\mathcal{R}$ ). On the other hand, let $V=] \alpha, \beta[\cap P$ be a basic open subset of $P$ (with $1<\alpha<\beta<2$ ), 
and let $\bar{x} \in V$ : choosing $\left.q^{\prime} \in Q \cap\right] 1 / \beta, 1 / \bar{x}\left[\right.$ and $\left.q^{\prime \prime} \in Q \cap\right] \alpha, \bar{x}$, we have that $A_{\bar{x}} \in\left[q^{\prime} / 1\right]_{d} \cap\left[q^{\prime \prime} / 0\right]_{d} \cap \mathcal{R} \subseteq\left\{A_{x} \mid x \in V\right\}$.

Consequently, it is clear that the Borel sets of $\mathcal{R}$ are exactly those of the form $\left\{A_{x} \mid x \in B\right\}$, with $B$ Borel in $P$. Since the Borel sets of $P$ do not coincide with the whole of $\wp(P)$, we also have that the Borel sets of $\mathcal{R}$ do not coincide with the whole of $\wp(\mathcal{R})$.

Second, consider the metric $\rho$ on $Q \cup P$ defined by:

$$
\rho(x, y)= \begin{cases}0, & \text { if } x=y ; \\ 2, & \text { if either } x \in P \text { and } y \in A_{x}, \text { or } y \in P \text { and } x \in A_{y} \\ 1, & \text { otherwise. }\end{cases}
$$

Note that for $x, y \in P$ we have that $\rho\left(x, A_{y}\right)=2$ if and only if $x=y$ (because the family $\mathcal{R}$ is incomparable), so that $[x / 2]_{\rho} \cap \mathcal{R}=\left\{A_{x}\right\}$. It follows that $\left(c_{0}(Q \cup P), \mathbf{w}_{\rho}\right)$ induces the discrete topology on $\mathcal{R}$, and hence the Borel sets of $\mathcal{R}$ with respect to such a topology are the whole of $\wp(\mathcal{R})$.

Since the Borel sets of a subspace are the traces of the Borel sets of the whole space, we also have that $\Xi_{d} \neq \Xi_{\rho}$.

\section{ACKNOWLEDGMENT}

The author wishes to thank S. Levi for pointing out to him the second problem tackled in this paper. He is also extremely grateful to the referee, for some essential improvements which have largely simplified and shortened the construction of both the examples.

\section{REFERENCES}

[Be1] G. Beer, Topologies on closed and closed convex sets, Kluwer Academic Publishers, Dordrecht, 1993. MR 95k:49001

[Be2] G. Beer, A Polish topology for the closed subsets of a Polish space, Proc. Amer. Math. Soc. 113 (1991), 1123-1133. MR 92c:54009

[CLZ] C. Costantini, S. Levi and J. Zieminska, Metrics that generate the same hyperspace convergence, Set-Valued Anal. 1 (1993), 141-157. MR 94h:54010

[Co] C. Costantini, Every Wijsman topology relative to a Polish space is Polish, Proc. Amer. Math. Soc. 123 (1995), 2569-2574. MR 95j:54012

[En] R. Engelking, General Topology, revised and completed edition, Heldermann Verlag, Berlin, 1989. MR 91c:54001

[He] C. Hess, Loi de la probabilité et indépendence des ensembles aléatoires à valeurs fermées dans un espace de Banach, Séminaire d'Analyse Convexe de l'Université de Montpellier, Exposé 7, 1983. MR 85m:60020

[Ke] A. S. Kechris, Classical Descriptive Set Theory, Springer-Verlag, New York, 1995. MR 96e:03057

[Zs] L. Zsilinszky, Baire spaces and hyperspace topologies, Proc. Amer. Math. Soc. 124 (1996), 2575-2584. MR 96j:54017

Dipartimento di Matematica, Università di Torino, Via Carlo Alberto 10, 10123 TORINO, ITALY

E-mail address: costantini@dm.unito.it 